ARTICLE

DOI: $10.1038 / \mathrm{s} 41467-018-03757-0$

\title{
Origin of vertical orientation in two-dimensional metal halide perovskites and its effect on photovoltaic performance
}

Alexander Z. Chen ${ }^{1}$, Michelle Shiu', Jennifer H. Ma', Matthew R. Alpert ${ }^{1}$, Depei Zhang ${ }^{2}$, Benjamin J. Foley ${ }^{1}$, Detlef-M. Smilgies (i) ${ }^{3}$, Seung-Hun Lee ${ }^{2} \&$ Joshua J. Choi (1) ${ }^{1}$

Thin films based on two-dimensional metal halide perovskites have achieved exceptional performance and stability in numerous optoelectronic device applications. Simple solution processing of the 2D perovskite provides opportunities for manufacturing devices at drastically lower cost compared to current commercial technologies. A key to high device performance is to align the $2 \mathrm{D}$ perovskite layers, during the solution processing, vertical to the electrodes to achieve efficient charge transport. However, it is yet to be understood how the counter-intuitive vertical orientations of 2D perovskite layers on substrates can be obtained. Here we report a formation mechanism of such vertically orientated 2D perovskite in which the nucleation and growth arise from the liquid-air interface. As a consequence, choice of substrates can be liberal from polymers to metal oxides depending on targeted application. We also demonstrate control over the degree of preferential orientation of the 2D perovskite layers and its drastic impact on device performance.

\footnotetext{
${ }^{1}$ Department of Chemical Engineering, University of Virginia, Charlottesville, VA 22904, USA. ${ }^{2}$ Department of Physics, University of Virginia, Charlottesville, VA 22904, USA. ${ }^{3}$ Cornell High Energy Synchrotron Source, Cornell University, Ithaca, NY 14853, USA. Correspondence and requests for materials should be addressed to J.J.C. (email: jjc6z@virginia.edu)
} 
M etal halide perovskites (MHPs) are poised to revolutionize the field of optoelectronic materials with their phenomenal performance advancement in solar cells ${ }^{1-}$ 5 , light-emitting diodes (LEDs) ${ }^{6-9}$, photodetectors ${ }^{10-14}$, and lasers ${ }^{15-17}$. MHPs are unique in that they combine low-cost solution processability with superb electronic quality that is comparable to, or surpasses, that of the state-of-the-art epitaxial grown semiconductors ${ }^{18-21}$. Moreover, MHPs enable lightweight flexible device applications due to the fact that they can be deposited on various substrates at low temperature $\left(<150^{\circ} \mathrm{C}\right)^{22-}$ 25

Despite their enormous potential, instability of MHPs is currently a major challenge to their device applications. Recently, two-dimensional (2D) Ruddlesden-Popper MHPs have been identified as materials that can potentially combine highperformance and long-term stability ${ }^{26-31}$. Going from threedimensional (3D) perovskite $A M X_{3} \quad\left(A=\mathrm{CH}_{3} \mathrm{NH}_{3}{ }^{+}, \mathrm{HC}\right.$ $\left.\left(\mathrm{NH}_{2}\right)_{2}{ }^{+}, \mathrm{Cs}^{+}, \mathrm{Rb}^{+} ; M=\mathrm{Sn}^{2+}, \mathrm{Pb}^{2+} ; X=\mathrm{Cl}^{-}, \mathrm{Br}^{-}, \mathrm{I}^{-}\right)$to $2 \mathrm{D}$ $B_{2} A_{n-1} M_{n} X_{3 n+1}\left(B=\mathrm{R}-\mathrm{NH}_{2}{ }^{+}\right)$, bulky long-chain ammonium cations are introduced to confine layers of metal halide octahedra in two dimensions. 2D MHPs exhibit many of the superb optoelectronic properties of their 3D counterparts while providing an opportunity to tune the properties by controlling the thickness of the metal halide layers through the quantum confinement $\mathrm{effect}^{32}$. Due to the bulky long-chain surface terminating ligands that are hydrophobic, 2D MHPs show drastically improved stability against humidity compared to $3 \mathrm{D} \mathrm{MHPs}^{26-31}$.

A critical requirement for high-performance 2D MHP optoelectronic devices is to align the $2 \mathrm{D}$ MHP layers perpendicular to the electrode layers. This is because the bulky ammonium cations that separate the 2D metal halide slabs are electrically insulating. For devices such as solar cells and LEDs, wherein the MHP thin films are sandwiched between electrodes, vertical alignment of 2D MHPs is desired to obtain efficient charge transport to the electrodes ${ }^{27}, 28$. However, the self-assembly processes through which $2 \mathrm{D}$ MHPs can be aligned vertically remain a mystery and cannot yet be controlled.

Here we reveal a mechanism responsible for the formation of vertically orientated $2 \mathrm{D}$ MHPs in thin films using grazing incidence wide angle X-ray scattering (GIWAXS). The thin films of $\mathrm{BA}_{2} \mathrm{MA}_{3} \mathrm{~Pb}_{4} \mathrm{I}_{13} \quad(\mathrm{BA}=$ butylammonium, $\quad \mathrm{MA}=$ methylammonium) are fabricated on various substrates using a simple solution processing at a fixed low temperature between 25 and $140{ }^{\circ} \mathrm{C}$. Our ex situ GIWAXS results show different degrees of preferential vertical orientation depending on the processing conditions. Solar cell performance measurements show that $\mathrm{BA}_{2} \mathrm{MA}_{3} \mathrm{~Pb}_{4} \mathrm{I}_{13}$ with a higher degree of vertical orientation results in drastically higher power conversion efficiency than those with more random orientations. In order to understand how the vertical orientation forms, we have performed systematically designed in situ GIWAXS experiments. Most importantly, our results show that heterogeneous nucleation and growth of $\mathrm{BA}_{2} \mathrm{MA}_{3} \mathrm{~Pb}_{4} \mathrm{I}_{13}$ occur at the liquid-air interface to form a top-crust with strong vertical orientation. We confirm this by repeating the GIWAXS measurements before and after selectively removing the top crust. The formation of vertically orientated 2D MHPs at the anisotropic environment of the liquid-air interface is likely due to preference for the aliphatic chain of butylammonium molecules bound to the metal halide slabs to remain in the solution environment. Knowing the origin of the preferential orientation allows us to rationally tune the degree of vertical orientation of $2 \mathrm{D}$ MHP with similar thin film morphology. As a consequence of the top-down growth mechanism, high-quality $2 \mathrm{D}$ MHP thin films can be deposited on various substrates ranging from polymers to metal oxides.
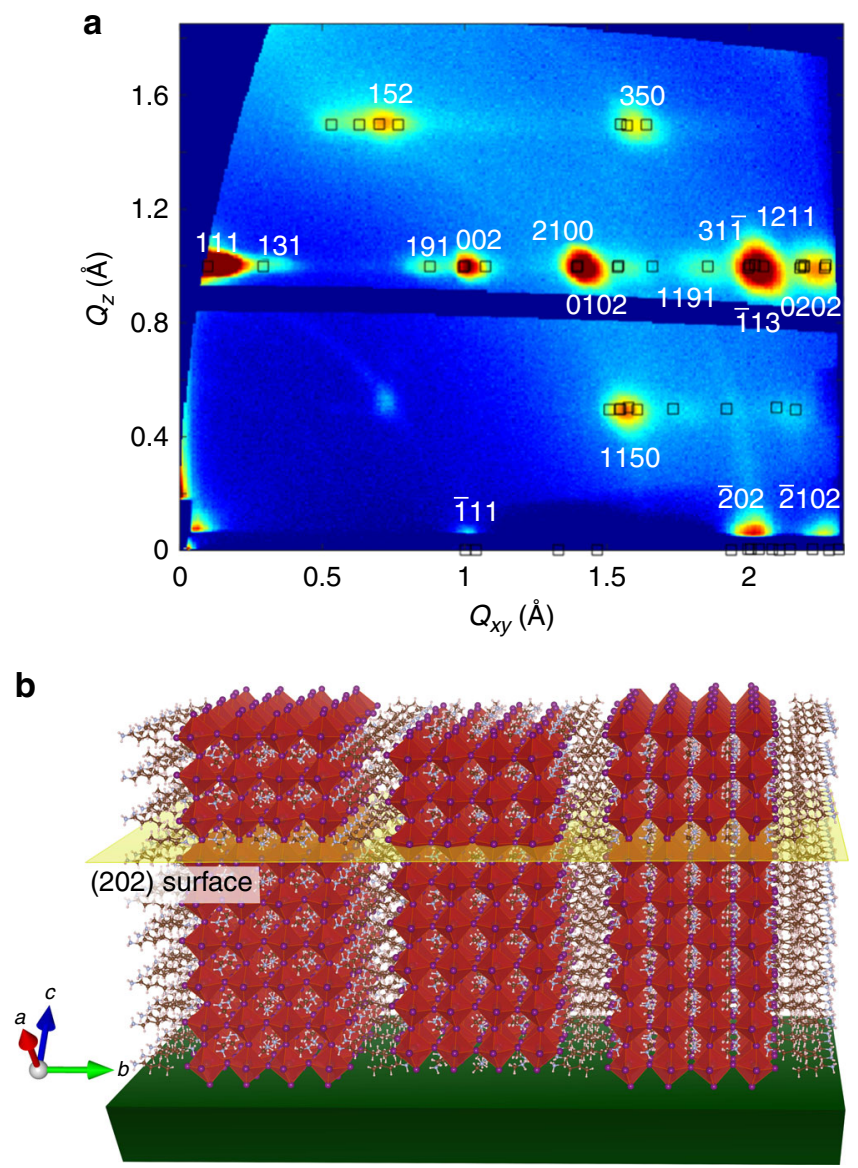

Fig. 1 GIWAXS pattern with indexing and illustration of the crystallographic orientation. a GIWAXS pattern of a $\mathrm{BA}_{2} \mathrm{MA}_{3} \mathrm{~Pb}_{4} \mathrm{l}_{13}$ thin film spin-coated from DMAc solution. The experimental peak positions agree well with simulation (black square) from an orthorhombic (101) vertically oriented $\mathrm{BA}_{2} \mathrm{MA}_{3} \mathrm{~Pb}_{4} \mathrm{l}_{13}$ structure. $\mathbf{b}$ Illustration of an orthorhombic (101) vertically oriented 2D perovskite structure, with (202) planes parallel to the substrate

\section{Results}

Structural and optical characterization. $\mathrm{BA}_{2} \mathrm{MA}_{3} \mathrm{~Pb}_{4} \mathrm{I}_{13}$ films were synthesized using a simple method of sequential spincoating and thermal annealing steps (see Methods section for details). The precursor solution was prepared by dissolving stoichiometric amounts of lead iodide $\left(\mathrm{PbI}_{2}\right)$, methylammonium iodide (MAI), and butylammonium iodide (BAI) in dimethylacetamide (DMAc) solvent. DMAc was chosen because it suppresses formation of intercalated intermediate structures leading to smooth morphology ${ }^{33}$. The solution was spin-coated onto a substrate briefly and, before the liquid film dried and turned dark, the sample was transferred to a hotplate set at various temperatures ranging from 25 to $140{ }^{\circ} \mathrm{C}$ (Supplementary Fig. 2). The MHP nucleation and growth then occur on the hotplate and result in a dark colored thin film. Our DMAc spin-coating method yields a uniform and reflective surface with a complete coverage, as evidenced by the scanning electron microscope (SEM) image shown in Supplementary Fig. 1c. The XRD pattern from the $\mathrm{BA}_{2} \mathrm{MA}_{3} \mathrm{~Pb}_{4} \mathrm{I}_{13}$ film indicates an orthorhombic (101) vertical crystallographic orientation with respect to the substrate $^{27,28}$ as shown in Supplementary Fig. 3b. The bandgap energy was measured to be $1.67 \mathrm{eV}$ using absorbance and photoluminescence spectroscopy (Supplementary Fig. $1 \mathrm{~b}$ and 4) which is consistent with the previous literature ${ }^{28,34}$. Excitonic peaks at locations consistent with lower layer $(n<4)$ impurities 
a
Oriented perovskite grown on $\mathrm{mp}-\mathrm{TiO}_{2}$ substrate

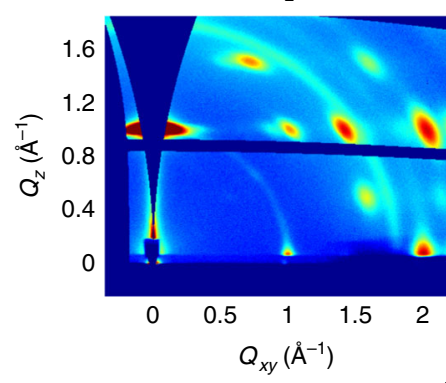

Nucleating within liquid

C film-random orientation

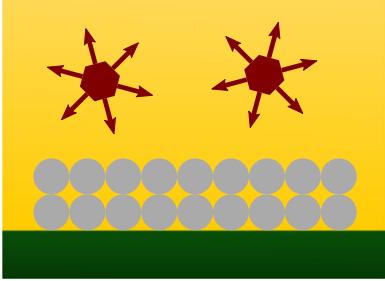

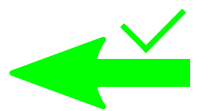

b

Nucleating from air-liquid interface-preferential orientation

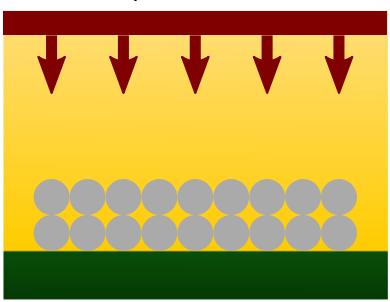

Nucleating from liquid-substrate

d interface-random orientation

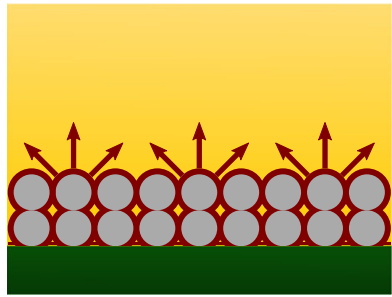

Fig. 2 Possible scenarios for nucleation with mesoporous $\mathrm{TiO}_{2}$ substrates. a GIWAXS pattern of $\mathrm{BA}_{2} \mathrm{MA}_{3} \mathrm{~Pb}_{4} \mathrm{l}_{13}$ thin film formed on mesoporous TiO 2 (mp$\mathrm{TiO}_{2}$ ) substrates indicates a strong vertical orientation. The continuous ring at $1.8 \AA$ is diffraction signal from mp-TiO ${ }_{2}$ substrate. b-d Illustration of possible crystallization processes from three different nucleation sites: liquid-air interface $\mathbf{b}$, within bulk liquid $\mathbf{c}$, and substrate-liquid interface $\mathbf{d}$. The gray circle stacks represent $\mathrm{mp}-\mathrm{TiO}_{2}$ substrate, the brown species represent $\mathrm{BA}_{2} \mathrm{MA}_{3} \mathrm{~Pb}_{4} \mathrm{l}_{13}$ and the brown arrows represent the crystallization direction. With the $\mathrm{mp}-\mathrm{TiO}_{2}$ substrate, only the nucleation and growth from the liquid-air interface scenario is consistent with formation of a vertically oriented $\mathrm{BA}_{2} \mathrm{MA}_{3} \mathrm{~Pb}_{4} \mathrm{I}_{13}$ thin film

are also present in absorption spectrum (Supplementary Fig. 1b), as typically observed in solution processed $\mathrm{BA}_{2} \mathrm{MA}_{3} \mathrm{~Pb}_{4} \mathrm{I}_{13}$ thin films $8,9,35,36$. For comparison with our DMAc method, we have also fabricated $\mathrm{BA}_{2} \mathrm{MA}_{3} \mathrm{~Pb}_{4} \mathrm{I}_{13}$ thin films using previously reported methods such as dimethylformamide (DMF)-based spin-coating 27 and hot casting ${ }^{28}$. In the "hot-casting" process, precursor solution with DMF as a solvent is casted on a hot spinning substrate to crystalize rapidly ${ }^{28}$. Detailed fabrication procedures and characterization results of these films are described in Methods section and Supplementary Fig. 3 and 5, respectively.

To obtain more in-depth structural information on the $\mathrm{BA}_{2} \mathrm{MA}_{3} \mathrm{~Pb}_{4} \mathrm{I}_{13}$ thin films, grazing incidence wide angle $\mathrm{X}$-ray scattering (GIWAXS) was performed on our samples ${ }^{37-39}$ (see Methods section for details). In the 2D GIWAXS patterns, diffraction intensity concentrated in spots indicates preferential crystallographic orientation with respect to the substrate while, in contrast, the diffraction intensity spread out into rings indicates randomly oriented crystals 37,38 . A GIWAXS pattern from a $\mathrm{BA}_{2} \mathrm{MA}_{3} \mathrm{~Pb}_{4} \mathrm{I}_{13}$ thin film prepared with our DMAc method is shown in Fig. 1a. The diffraction intensity is highly concentrated in spots, indicating a high degree of preferential crystallographic orientation. The pattern can be well indexed with a calculated pattern from the structure of $\mathrm{BA}_{2} \mathrm{MA}_{3} \mathrm{~Pb}_{4} \mathrm{I}_{13}{ }^{40}$ with (202) planes parallel to the substrate. Line-cuts of the GIWAXS pattern are consistent with the calculated pattern as shown in Supplementary Fig. 6 . The crystallographic structure determined by our GIWAXS results is illustrated in Fig. $1 \mathrm{~b}$ that shows vertically oriented $\mathrm{BA}_{2} \mathrm{MA}_{3} \mathrm{~Pb}_{4} \mathrm{I}_{13}$.

Nucleation and growth mechanism of vertical orientation. It is intriguing how the strong vertical orientation can form from solution through the spin-coating-annealing process. Homogeneous nucleation within the bulk of the solution results in growth of randomly oriented crystals due to the isotropic environment ${ }^{41,42}$. In certain cases, crystals from homogeneous nucleation can result in a preferential orientation upon deposition on a substrate if the crystal has anisotropic dimensions and has a preferential orientation that maximizes interactions between the crystals and the substrate, such as van der Waals (vdW) attraction. However, such a case typically induces horizontal, rather than vertical, orientation of the $2 \mathrm{D}$ crystal plates to maximize the vdW attraction ${ }^{43}$. Therefore, the observed strong vertical orientation in our films suggest that homogeneous nucleation is suppressed and, instead, heterogeneous nucleation at an interface is dominant. The question that arises is at which interface the heterogeneous nucleation occurs, the substrate-liquid interface, liquid-air interface or both? Finding the answer to this question is crucial in understanding the formation mechanism of vertically oriented the 2D MHP thin films that are required for high performance devices.

First, to check if the nucleation occurs at the substrate-liquid interface, we self-assembled $\mathrm{BA}_{2} \mathrm{MA}_{3} \mathrm{~Pb}_{4} \mathrm{I}_{13}$ thin films using our DMAc method on various different substrates including PEDOT, $\mathrm{SnO}_{2}, \mathrm{NiO}_{x}, \mathrm{TiO}_{x}$, and mesoporous $\mathrm{TiO}_{2}$. We found that strong vertical orientation of $\mathrm{BA}_{2} \mathrm{MA}_{3} \mathrm{~Pb}_{4} \mathrm{I}_{13}$ on all of these substrates (Supplementary Fig. 7). It is particularly striking that highly oriented $\mathrm{BA}_{2} \mathrm{MA}_{3} \mathrm{~Pb}_{4} \mathrm{I}_{13}$ is obtained even on mesoporous $\mathrm{TiO}_{2}$ substrate. If nucleation and subsequent growth happens at the liquid-substrate interface, we would expect the highly tortuous and rough surface of mesoporous $\mathrm{TiO}_{2}$ substrate to result in crystal growth in all directions (Fig. 2d), resulting in mostly random orientation of 2D MHP crystals. However, even on a mesoporous $\mathrm{TiO}_{2}$ substrate, the GIWAXS pattern shows a strong vertical orientation in the $\mathrm{BA}_{2} \mathrm{MA}_{3} \mathrm{~Pb}_{4} \mathrm{I}_{13}$ thin film (Fig. 2a). The ring at $q$ around $1.8 \AA^{-1}$ corresponds to diffraction intensity from the mesoporous $\mathrm{TiO}_{2}$ substrate at the bottom (Supplementary Fig. 8), indicating that the X-ray beam is probing all the way to the substrate surface. The lack of 


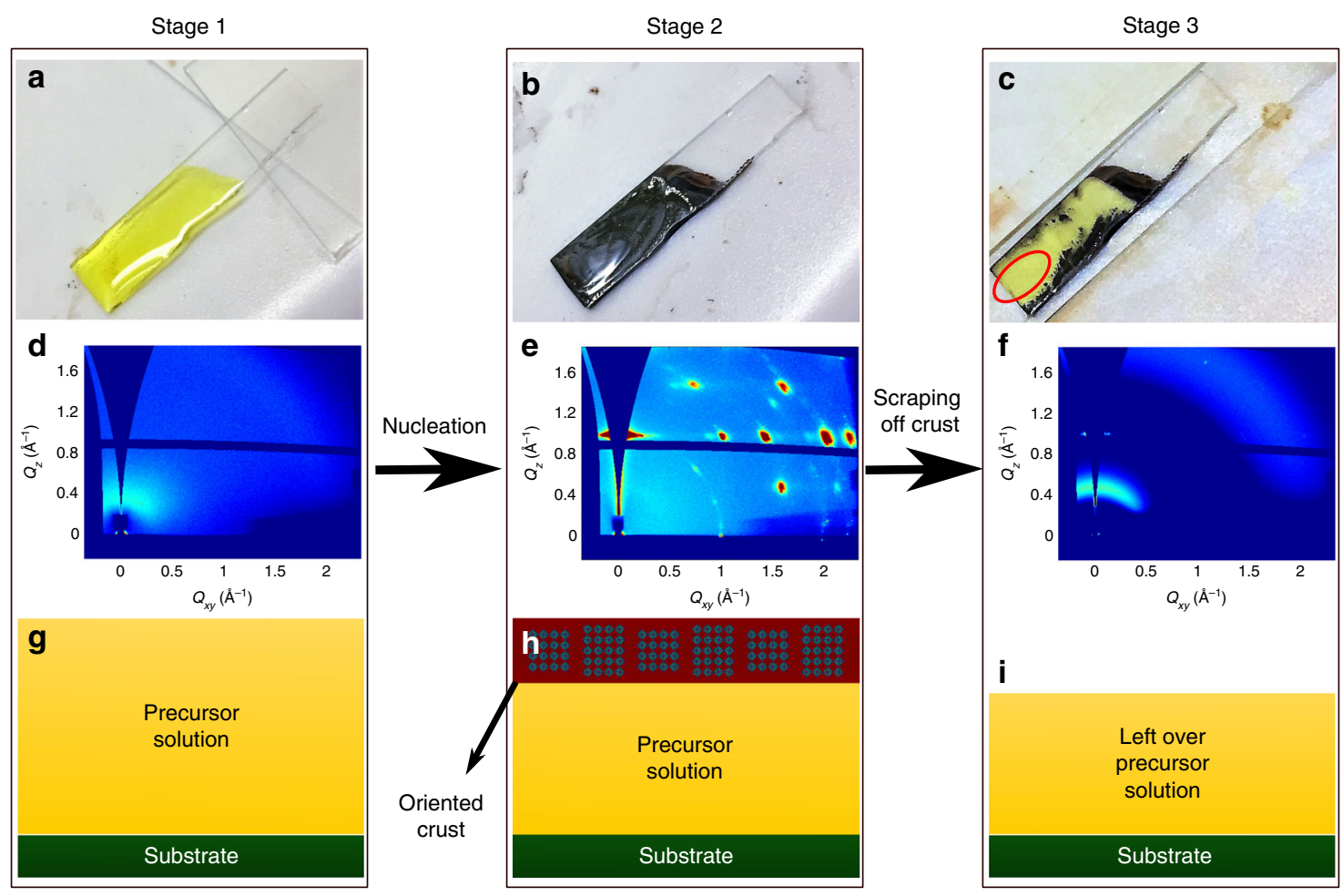

Fig. 3 Optical images and GIWAXS patterns and illustrations of top-crust scraping test. In stage 1, the precursor solution is deposited on a glass substrate. Optical image $\mathbf{a}$ and GIWAXS pattern $\mathbf{d}$ shows yellow liquid with no crystalline species $\mathbf{g}$. In stage 2, immediately after the surface turns dark upon thermal annealing $\mathbf{b}$, GIWAXS pattern e shows a vertically oriented $\mathrm{BA}_{2} \mathrm{MA}_{3} \mathrm{~Pb}_{4} \mathrm{I}_{13}$ top-crust $\mathbf{h}$. The top-crust is removed by a blade-scraping setup in stage 3 to expose the yellow precursor solution underneath that has not yet crystallized $\mathbf{c}$. GIWAXS pattern of the exposed spot $\mathbf{f}$ shows diffuse scattering only from its non-crystalline left-over precursor solution $\mathbf{i}$

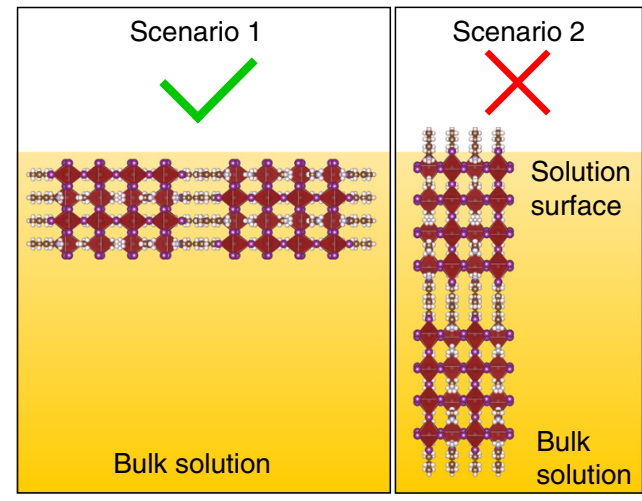

Fig. 4 Schematics of preferred orientation at liquid-air interface. The two possible nucleus orientations at the anisotropic precursor solution-air interface are shown in scenario 1 and scenario 2 . The vertical orientation in crystallized crust confirmed by GIWAXS indicates that scenario 1 is occurring

dependence on different substrates as well as the formation of strong vertical orientation regardless of the tortuous and uneven surface of the mesoporous $\mathrm{TiO}_{2}$ substrate indicates that the nucleation does not occur at the substrate-liquid interface. As discussed previously, nucleation and growth within the liquid bulk (Fig. 2c) are also expected to be absent as such a case would result in randomly oriented crystals due to the isotropic environment in the bulk solution, or horizontally oriented crystals upon deposition on the substrate. Based on these results, the most likely scenario is that the vertically oriented $\mathrm{BA}_{2} \mathrm{MA}_{3} \mathrm{~Pb}_{4} \mathrm{I}_{13}$ crystals originate from the anisotropic environment of liquid-air interface, regardless of the substrate choice as illustrated in Fig. 2 b.

Next, we have performed in situ GIWAXS experiments to check for the nucleation at the liquid-air interface. As illustrated in Fig. 2b, nucleation starting from the liquid-air interface can form a top-crust of highly oriented solid crystal film as a template for further crystal growth, with non-crystalline precursor solution underneath. To confirm this scenario, we designed a home-made blade setup (Supplementary Fig. 9) to selectively remove the topcrust during the self-assembly process in the following way. The precursor solution was deposited on a glass substrate (stage 1). The wet film was then annealed at $60^{\circ} \mathrm{C}$. Shortly after the whole film turned black (stage 2), the top-crust of the film was scraped off with a sharp blade (stage 3). The blade was configured to suspend above the substrate so that it did not touch the substrate surface during the process. GIWAXS patterns were taken at each stage to probe the presence of $\mathrm{BA}_{2} \mathrm{MA}_{3} \mathrm{~Pb}_{4} \mathrm{I}_{13}$. In stage 1 , the deposited precursor is a yellow liquid that has not yet crystallized (Fig. 3a), and the GIWAXS pattern shows no crystalline diffraction peaks in the wet film (Fig. 3d) and only shows diffuse scattering from the solution. The precursor liquid film was then annealed at $60^{\circ} \mathrm{C}$. Immediately after the film surface turned dark (Fig. 3b), in stage 2, the GIWAXS pattern shown in Fig. 3e indicates the formation of a vertically oriented crystalline $\mathrm{BA}_{2} \mathrm{MA}_{3} \mathrm{~Pb}_{4} \mathrm{I}_{13}$ film. However, when viewing from the other side of the glass substrate, liquid solution was observed (Supplementary Fig. 10), indicating that the precursor solution underneath the oriented perovskite crust has not yet crystallized. In stage 3, the perovskite top-crust was scraped off with a blade. The scraping of the top-crust exposed the yellow precursor solution underneath (Fig. 3c). GIWAXS measurement on the scraped spot (Fig. 3f) showed diffuse scattering from the liquid only with no 

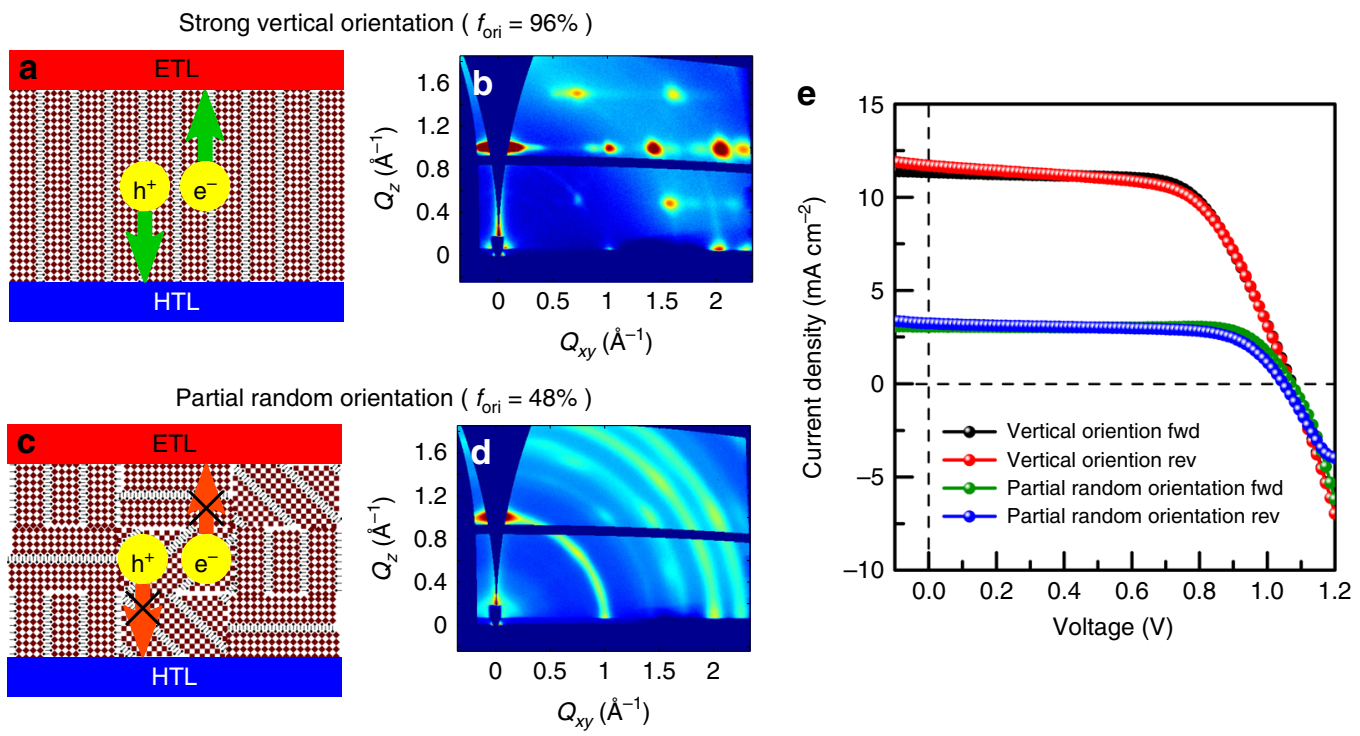

Fig. 5 Charge transport and device performance with different degrees of orientation. Pre-crystallization annealed film with strong vertical orientation indicated by GIWAXS in $\mathbf{b}$ provides direct pathway for electron and hole extraction in $\mathbf{a}$, while in post-crystallization annealed film with partial random orientation in $\mathbf{d}$, charge carriers need to hop through electrically insulating organic ligands to reach electron transporting layer (ETL) and hole transporting layer (HTL), which hinders charge extraction in c. The percentage of oriented crystallites in the perovskite film is defined as fori. Performance of solar cell with pre-crystallization annealing method (red and black lines) is significantly higher than that of post-crystallization annealing method (green and blue lines) in $\mathbf{e}$

crystalline peaks, confirming the visual observation. The fact that the diffuse scattering ring narrows and shifts to higher $q$ suggests that there could be changes in structure of precursors, but still without any long-range periodicity.

This test confirms that nucleation of $\mathrm{BA}_{2} \mathrm{MA}_{3} \mathrm{~Pb}_{4} \mathrm{I}_{13}$ occurs at the liquid-air interface. It has recently been reported that surface tension makes nucleation and growth at the liquid-air interface of perovskite precursor solution more favorable ${ }^{44}$. A similar process may be responsible for the $2 \mathrm{D}$ perovskite system as well. The confirmation of the crystallization at the liquid-air interface sheds light on the origin of the peculiar preferential vertical orientations. The orientation of the nucleus formed at the liquid-air interface and subsequent crystal growth determine the crystallographic orientation. Our observation of the vertically oriented $\mathrm{BA}_{2} \mathrm{MA}_{3} \mathrm{~Pb}_{4} \mathrm{I}_{13}$ thin film crystallizing from the liquid-air interface indicates that the initial nuclei are oriented in vertical configuration as illustrated in Fig. 4a, rather than the horizontal configuration as in Fig. 4b. It is possible that, with the butylammonium molecules bound to the lead iodide slabs such that the butyl chain are pointing toward outside, vertical orientation of the $2 \mathrm{D}$ perovskite nuclei is favored such that the butyl chains stay well inside the solution (Fig. 4a). The formation of vertically oriented $\mathrm{BA}_{2} \mathrm{MA}_{3} \mathrm{~Pb}_{4} \mathrm{I}_{13}$ also indicates that a monolayer of butylammonium molecules as typically seen in Langmuir-Blodgett films $s^{45}$ with butyl chains vertical to the liquid-air interface, likely does not form in this situation. If such a monolayer of butylammonium molecules formed at the liquid-air interface, it would have induced horizontal orientation of $\mathrm{BA}_{2} \mathrm{MA}_{3} \mathrm{~Pb}_{4} \mathrm{I}_{13}$. However, our observation of vertically oriented $\mathrm{BA}_{2} \mathrm{MA}_{3} \mathrm{~Pb}_{4} \mathrm{I}_{13}$ suggests that is not the case. It is likely that the butylammonium molecules are not able to form a monolayer at the liquid-air interface due to either DMAc-butylammonium-air interaction does not favor formation of monolayer thin film, or a majority of butylammonium complexing with lead iodide species.

Controlling degree of vertical orientation. Knowing the origin of the preferential orientation allows us to rationally control the degree of orientation by tuning the crystallization conditions. We refer to the crystallization process described so far as "pre-crystallization annealing" where the $\mathrm{BA}_{2} \mathrm{MA}_{3} \mathrm{~Pb}_{4} \mathrm{I}_{13}$ film crystallizes on a hotplate at elevated temperature with minimal spin-induced convection. In contrast, if the precursor solution film is left for a longer period during spin-coating process such that the film gets dried while spinning, the evaporation rate of the solvent can be kept high with spin-induced convection while the lack of heating keeps the diffusion rates of precursor species low. This can induce a significant amount of homogeneous nucleation throughout the solution which will result in more randomly oriented $\mathrm{BA}_{2} \mathrm{MA}_{3} \mathrm{~Pb}_{4} \mathrm{I}_{13}$. The thermal annealing is then applied after the crystallization has been completed during spinning. We call this procedure as "post-crystallization annealing" method. Figure 5b, d shows that the films made using the two different methods show very different degrees of the preferential vertical orientation. Figure $5 \mathrm{~b}$ shows that the pre-crystallization annealed film shows a degree of preferential vertical orientation, $f_{\text {ori }}$, of $96 \%$. Fig $5 \mathrm{~d}$, on the other hand, shows that the post-crystallization annealed film has $f_{\text {ori }}$ of $48 \%$, which means that about half of the $\mathrm{BA}_{2} \mathrm{MA}_{3} \mathrm{~Pb}_{4} \mathrm{I}_{13}$ plates are randomly oriented. The details regarding quantification of $f_{\text {ori }}$ is available in Method section, Supplementary Fig. 11 and Supplementary Table 1 . Although they are fabricated from the same precursor, substrate, spin speed, and glovebox environment, with similar film morphology (Supplementary Fig. 12), different thermal annealing timing results in drastically different degree of preferential orientation.

Impact of orientation on solar cell performance. The control over the degree of preferential orientation in $\mathrm{BA}_{2} \mathrm{MA}_{3} \mathrm{~Pb}_{4} \mathrm{I}_{13}$ thin films enabled us to systematically compare its effect on solar cell performance. It is expected that a vertically oriented $2 \mathrm{D}$ perovskite thin film provides direct pathway for electron and hole transport, while the charge transport is hindered by hopping across the long-chain organic surface ligands in a more randomly oriented film (Fig. 5a, c). To test this, we fabricated and characterized $\mathrm{BA}_{2} \mathrm{MA}_{3} \mathrm{~Pb}_{4} \mathrm{I}_{13}$ solar cells using the pre- and postcrystallization annealing methods. The structure of solar cells was 
Table 1 Solar cell parameters of the pre- and post-crystallization annealed devices

\begin{tabular}{|c|c|c|c|c|c|c|c|}
\hline Method & Scanning direction & $V_{\text {oc }}(V)$ & $J_{\mathrm{sc}}\left(\mathrm{mA} \mathrm{cm} \mathrm{cm}^{-2}\right)$ & $\mathbf{F F}$ & $R_{\text {series }}\left(\Omega \mathrm{cm}^{2}\right)$ & $R_{\text {shunt }}\left(\Omega \mathrm{cm}^{2}\right)$ & Efficiency (\%) \\
\hline \multirow{2}{*}{ Pre-crystallization annealing } & Fwd & 1.07 & 11.3 & 64.8 & 22.3 & 1653 & 7.9 \\
\hline & $\operatorname{Rev}$ & 1.07 & 11.7 & 61.8 & 21.7 & 594 & 7.7 \\
\hline Post-crystallization annealing & Fwd & 1.07 & 3.0 & 78.7 & 36.0 & 7800 & 2.6 \\
\hline
\end{tabular}

ITO/PEDOT:PSS/BA $\mathrm{BA}_{3} \mathrm{~Pb}_{4} \mathrm{I}_{13} / \mathrm{PCBM} / \mathrm{Cu}$ (see Methods section for detailed procedure), and their performance is shown in Fig. 5e and Table 1. The device performance statistics based on 30 devices are shown in Supplementary Fig. 13. The $J-V$ curve of $\mathrm{BA}_{2} \mathrm{MA}_{3} \mathrm{~Pb}_{4} \mathrm{I}_{13}$ solar cells made with both methods show a slight hysteresis behavior, which is frequently observed in $3 \mathrm{D}$ perovskite solar cells, indicating mechanisms causing hysteresis in $3 \mathrm{D}$ perovskite solar cells also exist in 2D perovskite, including capacitive effect, trapping and detrapping of carriers, ion migration and ferroelectric effect ${ }^{46}$.

The pre-crystallization annealed film with a strong vertical orientation outperforms the films with partial random orientation due to a significantly higher short circuit current density $\left(J_{\mathrm{sc}}\right)$ and lower series resistance $\left(R_{\mathrm{s}}\right)$, while both devices have similar open circuit voltage $\left(V_{\text {oc }}\right)$. Both films have similar thicknesses of around $325 \mathrm{~nm}$, absorbance spectra and optical density (Supplementary Fig. 14 and 15). Therefore, the higher $J_{\text {sc }}$ of the precrystallization annealed solar cell is unlikely due to different amounts of light absorption. Shunt resistance $\left(R_{\mathrm{sh}}\right)$ from both set of devices have high values (Table 1), indicating the devices are not significantly affected by shunting from rough morphology and incomplete coverage. Combined together, these observations are consistent with the expectation of superior charge transport properties with vertical crystallographic orientation compared to randomly oriented 2D MHPs, which results in improved $J_{\text {sc }}$. Our results clearly show that a strong vertical orientation is necessary for achieving high performance in optoelectronic devices.

\section{Discussion}

In summary, we reveal that vertically oriented 2D MHP $\mathrm{BA}_{2} \mathrm{MA}_{3} \mathrm{~Pb}_{4} \mathrm{I}_{13}$ nucleates and grows from the precursor liquid-air interface. By comparing electronic properties of vertically oriented and partially randomly oriented thin films, we find that a strong vertical orientation is necessary for optimal solar cell performance. This insight on the 2D MHP self-assembly and its impact on optoelectronic device performance will accelerate the progress toward a wide-spread application of 2D MHPs devices.

\section{Methods}

Materials and instruments. PEDOT:PSS Al 4083 (Clevios) was filtered with a $0.45 \mu \mathrm{m}$ size filter before use. Methylammonium iodide (MAI) and butylammonium iodide (BAI) were purchased from Dyesol. $\mathrm{PbI}_{2}(99.9985 \%)$ was purchased from Alfa Aesar. Anhydrous dimethylacetamide (DMAc) (99.8\%), anhydrous DMF (99.8\%) and bathocuproine (BCP) (99.99\%) were purchased from Sigma Aldrich. Indium tin oxide (ITO) substrates $\left(15 \Omega \mathrm{cm}^{-2}\right)$ were purchased from Kintec. Phenyl-C61-butyric acid methyl ester (PCBM) (99.5\%) was purchased from NanoC. Copper pellets were purchase from Kurt Lesker.

X-ray diffraction. XRD was performed on a PANalytical X'pert system at $40 \mathrm{kV}$ and $40 \mathrm{~mA}$.

GIWAXS measurements and indexing method. GIWAXS was performed at D-1 beamline at Cornell High Energy Synchrotron Source (CHESS) using X-rays with a wavelength of $1.162 \AA$, a custom precision goniometer, and a Pilatus 200k 2D pixel array detector (Dectris). The temperature of the custom-built sample holder was controlled by a temperature controller (Digi-Sense) and the temperature was monitored during X-ray data collection. A typical X-ray incidence angle was $0.7^{\circ}$. The data reduction ${ }^{47}$ and calculation of the location and intensity of the diffraction peaks with PowderCell were performed using a previously reported structure ${ }^{40}$.
Perovskite film fabrication. Pre-crystallization annealing method: $0.75 \mathrm{M}$ $\mathrm{BA}_{2} \mathrm{MA}_{3} \mathrm{~Pb}_{4} \mathrm{I}_{13}$ solution in DMAc was made by dissolving $0.75 \mathrm{M} \mathrm{PbI}_{2}, 0.5625 \mathrm{M}$ MAI and $0.375 \mathrm{M}$ BAI with stirring. The solution was filtered with a $0.22 \mu \mathrm{m}$ filter and then deposited on the substrate by spinning at 2000 r.p.m. After about $20 \mathrm{~s}$ of spin-coating the slide was taken off the spincoater and immediately placed on a hotplate to anneal at $120^{\circ} \mathrm{C}$ for $10 \mathrm{~min}$, where the precursor crystallized and turned dark brown. Post-crystallization annealing method: $0.75 \mathrm{M} \mathrm{BA}_{2} \mathrm{MA}_{3} \mathrm{~Pb}_{4} \mathrm{I}_{13}$ solution dissolved in DMAc was filtered with a $0.22 \mu \mathrm{m}$ filter and then deposited on the substrate by spinning at 2000 r.p.m. After $60 \mathrm{~s}$ of spin-coating the slide had already dried and turned dark brown, and was taken off the spincoater and placed on a hotplate to anneal at $120^{\circ} \mathrm{C}$ for $10 \mathrm{~min}$.

Device fabrication and characterization. ITO slides were cleaned by sequentially sonication in soap water, deionized water and ethanol, then treated with ozone plasma for $10 \mathrm{~min}$ before use. PEDOT:PSS was deposited by spin-coating at $6000 \mathrm{r}$. p.m. for $60 \mathrm{~s}$, followed by annealing at $120^{\circ} \mathrm{C}$ for $15 \mathrm{~min}$. The slides were then transferred into a $\mathrm{N}_{2}$ filled glovebox. Pre-and-post-crystallization annealing methods were carried out to form the perovskite active layers, as described above. A layer of PCBM was deposited by spin-coating a freshly filtered $10 \mathrm{mg} \mathrm{ml}^{-1}$ solution in chloroform at 3000 r.p.m. for $60 \mathrm{~s}$, followed by annealing at $100^{\circ} \mathrm{C}$ for $10 \mathrm{~min}$. After the slides cooled down, a layer of BCP was spin-coated at 4000 r.p.m. with a $0.5 \mathrm{mg} \mathrm{ml}^{-1}$ solution in isopropanol. $50 \mathrm{~nm} \mathrm{Cu}$ electrode was then thermally evaporated in ultra-high vacuum $\left(<10^{-5}\right.$ torr $)$. Device active area is $0.03 \mathrm{~cm}^{2}$, defined by the overlap between ITO substrate and metal electrode, as well as an optical mask. The devices were tested under simulated Air Mass 1.5 irradiance of $100 \mathrm{~mW} \mathrm{~cm}^{-2}$ light source calibrated by a reference Si solar cell.

Calculating degree of preferential orientation. Bragg peaks $[2,0,0]$ and $[0,0,2]$ are fit with Gaussian distribution and the fitting results and coefficients are in Supplementary Fig. 13 and Supplementary Table 1. $\Delta Q_{\mathrm{rad}}=\Delta Q$ and $\Delta Q_{\tan }=$ $Q_{c} \times \Delta \chi$ are the fitted FWHMs in radial and tangential directions. The orientation correlation length $\xi_{\tan }$ is related to the tangential width of the Bragg peaks and can be calculated with the Scherrer equation, $\xi_{\tan }=\frac{2 \pi \times 0.9}{\sqrt{\left(\Delta Q_{\text {tan }}\right)^{2}-\left(\Delta Q_{\mathrm{res}}\right)^{2}}}$. The ratio of orientated crystallites, $f_{\text {ori }}=\frac{A_{\text {ori }}}{A_{\text {ori }}+A_{\text {iss }}}$, where $A_{\text {ori }}\left(A_{\text {iso }}\right)$ is the amount of orientated (isotropic) crystallites, is calculated with the area under the fitted Gaussian peak (without the flat line) divided by the total area under $\widetilde{I}(\chi)$ in Supplementary Fig. $11 b^{48}$."

Data availability. The data that support the findings of this study are available from the corresponding author upon request.

Received: 19 October 2017 Accepted: 8 March 2018

Published online: 06 April 2018

\section{References}

1. Kojima, A., Teshima, K., Shirai, Y. \& Miyasaka, T. Organometal halide perovskites as visible-light sensitizers for photovoltaic cells. J. Am. Chem. Soc. 131, 6050-6051 (2009).

2. Saliba, M. et al. Cesium-containing triple cation perovskite solar cells: improved stability, reproducibility and high efficiency. Energy Environ. Sci. 9, 1989-1997 (2016).

3. Yang, W. S. et al. High-performance photovoltaic perovskite layers fabricated through intramolecular exchange. Science 348, 1234-1237 (2015).

4. Bi, D. et al. Polymer-templated nucleation and crystal growth of perovskite films for solar cells with efficiency greater than 21\%. Nat. Energy 1, 16142 (2016).

5. Li, X. et al. A vacuum flash-assisted solution process for high-efficiency largearea perovskite solar cells. Science 353, 58-62 (2016).

6. Xiao, Z. et al. Efficient perovskite light-emitting diodes featuring nanometresized crystallites. Nat. Photonics 11, 108-115 (2017).

7. Byun, J. et al. Efficient visible quasi-2D perovskite light-emitting diodes. $A d v$ Mater. 28, 7515-7520 (2016). 
8. Yuan, M. et al. Perovskite energy funnels for efficient light-emitting diodes. Nat. Nanotechnol. 11, 872-877 (2016).

9. Wang, N. et al. Perovskite light-emitting diodes based on solution-processed self-organized multiple quantum wells. Nat. Photonics 10, 699-704 (2016).

10. Lin, Q., Armin, A., Lyons, D. M., Burn, P. L. \& Meredith, P. Low noise, IRblind organohalide perovskite photodiodes for visible light detection and imaging. Adv. Mater. 27, 2060-2064 (2015).

11. Dou, L. et al. Solution-processed hybrid perovskite photodetectors with high detectivity. Nat. Commun. 5, 5404 (2014).

12. Fang, Y. \& Huang, J. Resolving weak light of sub-picowatt per square centimeter by hybrid perovskite photodetectors enabled by noise reduction. Adv. Mater. 27, 2804-2810 (2015).

13. Yakunin, S. et al. Detection of X-ray photons by solution-processed lead halide perovskites. Nat. Photonics 9, 444-449 (2015).

14. Fang, Y., Dong, Q., Shao, Y., Yuan, Y. \& Huang, J. Highly narrowband perovskite single-crystal photodetectors enabled by surface-charge recombination. Nat. Photonics 9, 679-686 (2015).

15. Zhu, H. et al. Lead halide perovskite nanowire lasers with low lasing thresholds and high quality factors. Nat. Mater. 14, 636-642 (2015).

16. Eaton, S. W. et al. Lasing in robust cesium lead halide perovskite nanowires. Proc. Natl Acad. Sci. USA 113, 1993-1998 (2016).

17. Xing, G. et al. Low-temperature solution-processed wavelength-tunable perovskites for lasing. Nat. Mater. 13, 476-480 (2014).

18. Shi, D. et al. Solar cells. Low trap-state density and long carrier diffusion in organolead trihalide perovskite single crystals. Science 347, 519-522 (2015).

19. Yang, Y. et al. Observation of a hot-phonon bottleneck in lead-iodide perovskites. Nat. Photonics 10, 53-59 (2015).

20. Yin, W. J., Shi, T. \& Yan, Y. Unique properties of halide perovskites as possible origins of the superior solar cell performance. Adv. Mater. 26, 4653-4658 (2014).

21. Dong, Q. et al. Solar cells. Electron-hole diffusion lengths $>175$ mum in solution-grown $\mathrm{CH} 3 \mathrm{NH} 3 \mathrm{PbI} 3$ single crystals. Science 347, 967-970 (2015).

22. Deng, $\mathrm{X}$. et al. Room-temperature processing of $\mathrm{TiO}_{\mathrm{x}}$ electron transporting layer for perovskite solar cells. J. Phys. Chem. Lett. 8, 3206-3210 (2017).

23. Liu, D. \& Kelly, T. L. Perovskite solar cells with a planar heterojunction structure prepared using room-temperature solution processing techniques. Nat. Photonics 8, 133-138 (2013).

24. Ye, M., Hong, X., Zhang, F. \& Liu, X. Recent advancements in perovskite solar cells: flexibility, stability and large scale. J. Mater. Chem. A 4, 6755-6771 (2016).

25. Kaltenbrunner, M. et al. Flexible high power-per-weight perovskite solar cells with chromium oxide-metal contacts for improved stability in air. Nat. Mater. 14, 1032-1039 (2015).

26. Smith, I. C., Hoke, E. T., Solis-Ibarra, D., McGehee, M. D. \& Karunadasa, H. I. A layered hybrid perovskite solar-cell absorber with enhanced moisture stability. Angew. Chem. Int. Ed. 53, 11232-11235 (2014).

27. Cao, D. H., Stoumpos, C. C., Farha, O. K., Hupp, J. T. \& Kanatzidis, M. G. 2D homologous perovskites as light-absorbing materials for solar cell applications. J. Am. Chem. Soc. 137, 7843-7850 (2015).

28. Tsai, H. et al. High-efficiency two-dimensional Ruddlesden-Popper perovskite solar cells. Nature 536, 312-316 (2016)

29. Smith, I. C., Smith, M. D., Jaffe, A., Lin, Y. \& Karunadasa, H. I. Between the sheets: postsynthetic transformations in hybrid perovskites. Chem. Mater. 29, 1868-1884 (2017).

30. Slavney, A. H. et al. Chemical approaches to addressing the instability and toxicity of lead-halide perovskite absorbers. Inorg. Chem. 56, 46-55 (2017).

31. Mitzi, D. B., Medeiros, D. R. \& Malenfant, P. R. L. Intercalated organic -inorganic perovskites stabilized by fluoroaryl-aryl interactions. Inorg. Chem. 41, 2134-2145 (2002).

32. Kamminga, M. E. et al. Confinement effects in low-dimensional lead iodide perovskite hybrids. Chem. Mater. 28, 4554-4562 (2016).

33. $\mathrm{Lv}, \mathrm{M}$. et al. A promising alternative solvent of perovskite to induce rapid crystallization for high-efficiency photovoltaic devices. RSC Adv. 5, 20521-20529 (2015).

34. Blancon, J. C. et al. Extremely efficient internal exciton dissociation through edge states in layered 2D perovskites. Science 355, 1288-1292 (2017).

35. Milot, R. L. et al. Charge-carrier dynamics in $2 \mathrm{D}$ hybrid metal-halide perovskites. Nano Lett. 16, 7001-7007 (2016).

36. Liu, J., Leng, J., Wu, K., Zhang, J. \& Jin, S. Observation of internal photoinduced electron and hole separation in hybrid two-dimentional perovskite films. J. Am. Chem. Soc. 139, 1432-1435 (2017).

37. Foley, B. J. et al. Controlling nucleation, growth, and orientation of metal halide perovskite thin films with rationally selected additives. J. Mater. Chem. A 5, 113-123 (2017).
38. Chen, A. Z. et al. Crystallographic orientation propagation in metal halide perovskite thin films. J. Mater. Chem. A 5, 7796-7800 (2017).

39. Schlipf, J. \& Müller-Buschbaum, P. Structure of organometal halide perovskite films as determined with grazing-incidence X-ray scattering methods. $A d v$. Energy Mater. 7, 1700131 (2017).

40. Stoumpos, C. C. et al. Ruddlesden-Popper hybrid lead iodide perovskite 2D homologous semiconductors. Chem. Mater. 28, 2852-2867 (2016).

41. Porter, D. A. \& Easterling, K. E. Phase Transformations in Metals and Alloys, 2nd edn (Chapman \& Hall, London, 1992).

42. Zhou, Y., Game, O. S., Pang, S. \& Padture, N. P. Microstructures of organometal trihalide perovskites for solar cells: their evolution from solutions and characterization. J. Phys. Chem. Lett. 6, 4827-4839 (2015)

43. Ha, S. T., Su, R., Xing, J., Zhang, Q. \& Xiong, Q. Metal halide perovskite nanomaterials: synthesis and applications. Chem. Sci. 8, 2522-2536 (2017)

44. Zhumekenov, A. A. et al. The role of surface tension in the crystallization of metal halide perovskites. ACS Energy Lett. 2, 1782-1788 (2017)

45. Ariga, K., Yamauchi, Y., Mori, T. \& Hill, J. P. 25th anniversary article: what can be done with the Langmuir-Blodgett method? Recent developments and its critical role in materials science. Adv. Mater. 25, 6477-6512 (2013).

46. Chen, B., Yang, M., Priya, S. \& Zhu, K. Origin of $J-V$ hysteresis in perovskite solar cells. J. Phys. Chem. Lett. 7, 905-917 (2016).

47. Jiang, Z. GIXSGUI: a MATLAB toolbox for grazing-incidence X-ray scattering data visualization and reduction, and indexing of buried three-dimensional periodic nanostructured films. J. Appl. Crystallogr. 48, 917-926 (2015).

48. Oesinghaus, L. et al. Toward tailored film morphologies: the origin of crystal orientation in hybrid perovskite thin films. Adv. Mater. Interfaces 3, 1600403 (2016).

\section{Acknowledgements}

J.J.C. acknowledges support from an Early Career Faculty Award grant from NASA's Space Technology Research Grants Program (NNX15AU43G). S.-H.L. and J.J.C. acknowledge support from the US Department of Energy, Office of Science, Office of Basic Energy Sciences under Award Number DE-SC0016144. This work is based upon research conducted at the Cornell High Energy Synchrotron Source (CHESS), which is supported by the National Science Foundation under award DMR-1332208.

\section{Author contributions}

A.Z.C. and J.J.C. conceived and designed the research. A.Z.C., M.S., J.H.M., M.R.A., D.Z., B.J.F. D.-M.S., S.-H.L., and J.J.C. performed the experiments and data analysis. A.Z.C., S.H.L. and J.J.C. wrote the manuscript. All authors read and approved the manuscript.

\section{Additional information}

Supplementary Information accompanies this paper at https://doi.org/10.1038/s41467018-03757-0.

Competing interests: The authors declare no competing interests.

Reprints and permission information is available online at http://npg.nature.com/ reprintsandpermissions/

Publisher's note: Springer Nature remains neutral with regard to jurisdictional claims in published maps and institutional affiliations.

Open Access This article is licensed under a Creative Commons Attribution 4.0 International License, which permits use, sharing, adaptation, distribution and reproduction in any medium or format, as long as you give appropriate credit to the original author(s) and the source, provide a link to the Creative Commons license, and indicate if changes were made. The images or other third party material in this article are included in the article's Creative Commons license, unless indicated otherwise in a credit line to the material. If material is not included in the article's Creative Commons license and your intended use is not permitted by statutory regulation or exceeds the permitted use, you will need to obtain permission directly from the copyright holder. To view a copy of this license, visit http://creativecommons.org/ licenses/by/4.0/.

(c) The Author(s) 2018 